\title{
Nature, idéal et caricature. La perception des types physiques chez les premiers anthropologues
}

\section{Martial Guédron}

\section{(2) OpenEdition \\ 12 Journals}

Édition électronique

URL : http://journals.openedition.org/actesbranly/262

DOI : $10.4000 /$ actesbranly.262

ISSN : 2105-2735

Éditeur

Musée du quai Branly Jacques Chirac

Référence électronique

Martial Guédron, « Nature, idéal et caricature. La perception des types physiques chez les premiers anthropologues ", Les actes de colloques du musée du quai Branly Jacques Chirac [En ligne], 1 | 2009, mis en ligne le 28 juillet 2009, consulté le 07 septembre 2020. URL : http://journals.openedition.org/ actesbranly/262 ; DOI : https://doi.org/10.4000/actesbranly.262

Ce document a été généré automatiquement le 7 septembre 2020

(c) Tous droits réservés 


\title{
Nature, idéal et caricature. La perception des types physiques chez les premiers anthropologues
}

\author{
Martial Guédron
}

1 Il y a plusieurs décennies déjà, les travaux d'Ernst Hans Gombrich ont établi qu'il n'existe pas « d'œil innocent » et que nous sommes enclins à différencier, hiérarchiser et classer tout ce que nous voyons dans des catégories vérifiées par l'expérience. Convaincu que nous ne nous débarrassons jamais de ce que nous savons ou croyons savoir, Gombrich a montré comment les préjugés esthétiques s'insinuent dans la contemplation des objets artistiques comme dans celle de notre environnement. Analysant la manière dont nous percevons les paysages naturels, il a observé que la nature ne nous serait jamais apparue "pittoresque» si nous n'avions pas contracté l'habitude de la regarder comme une peinture, ou tout au moins à travers des codes mis au point par des peintres ${ }^{1}$. Que certains de ces codes interfèrent aussi dans la perception de la variété de l'espèce humaine, les textes fondateurs de l'anthropologie publiés en France à la charnière du XVII et $d u x^{e} x^{e}$ siècle en témoignent de manière éloquente. Mais il ne faut pas négliger le rôle des images dans cette affaire : c'est par l'interaction entre les images et les discours que les prescriptions esthétiques ont pesé de tout leur poids sur le déterminisme racial et l'échelonnement du genre humain.

\section{Profiler}

Rappelons à grands traits qu'à la fin du siècle des Lumières, les théories esthétiques concernant les représentations du corps humain étaient encore marquées par les écrits de Winckelmann. Dans le sillage de l'archéologue allemand, pour qui la sculpture grecque classique était la manifestation de la beauté absolue, Quatremère de Quincy prétendait ainsi que les figures antiques incarnaient des proportions immuables où l'on pouvait puiser tous les principes du beau et tous les éléments de la perfection ${ }^{2}$. On se souvient que dans ses descriptions des différents types humains, Winckelmann se 
fondait sur le profil grec, un critère auquel il donnait une valeur absolue : cette ligne presque droite passant par le front et le nez était tout juste marquée par une très douce inflexion. Plus les accents séparant le nez du front étaient prononcés, plus le profil paraissait disgracieux et s'éloignait de la belle forme. C'est ce qu'attestaient les yeux obliques des Chinois et des Japonais, le nez écrasé des Kalmouks et, par-dessus tout, la bouche charnue des Maures, cette excroissance qu'ils partageaient avec les singes de leur pays et qui était due à l'excès de chaleur de leur climat, à l'abondance de leurs humeurs âcres et à leur tempérament colérique ${ }^{3}$. On connaît les liens passionnants que l'histoire de l'art winckelmannienne a noués avec l'histoire naturelle de son siècle : sa conception de l'homme grec idéal y trouve en grande partie son origine ${ }^{4}$. Mais ce n'est pas tout.

Fig. 1. Petrus Camper, « La ligne facial du singe à queue, de l'orang-outang, du nègre et du kalmouk ", Dissertation physique sur les différences que présentent les traits du visage, Utrecht, 1791.

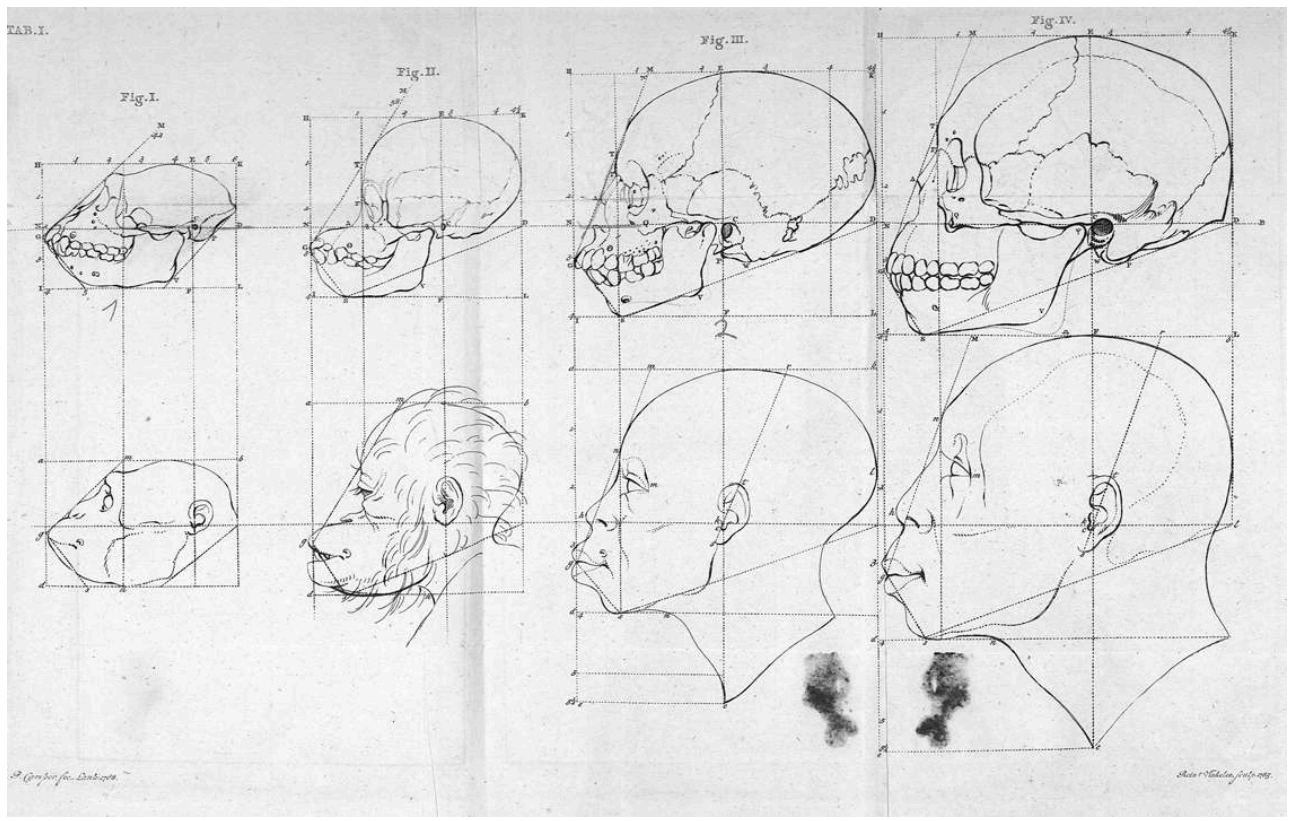

Photo : Bibliothèque interuniversitaire de médecine

Autour de 1800, en ce moment « naturaliste » des sciences de l'homme ${ }^{5}$, l'appréhension des différentes variétés humaines selon des critères esthétiques débordait le champ de l'histoire de l'art. Nombre de scientifiques partageaient avec les théoriciens néoclassiques l'idée que la beauté procédait directement des proportions. Comme on sait, leur argumentation s'appuyait sur le modèle des statues antiques, voire sur des illustrations qui accordaient une place très importante à la tête, celle-ci étant considérée comme la clé modulaire déterminant les proportions de l'ensemble du corps. Pendant que les écrits de Winckelmann suscitaient en France des comptes rendus, des articles et plusieurs traductions, l'anatomiste néerlandais Petrus Camper multipliait les démonstrations sur sa théorie de l'angle facial. Le 26 juillet 1777, lors d'un séjour à Paris, il prononçait une conférence sur ce thème devant l'Académie royale des sciences ${ }^{6}$, montrant à l'occasion de réelles dispositions pour le dessin, ce que confirment d'ailleurs ses contemporains ${ }^{7}$. Pour parvenir à une représentation aussi précise que possible du crâne vu de profil, il estimait que la technique la plus appropriée était celle du dessin par projection orthographique. À cette fin, il 
préconisait l'emploi d'un dispositif constitué d'une grille verticale composée de fils entrecroisés qui correspondaient à un quadrillage tracé sur la feuille de papier où l'on voulait dessiner le modèle ${ }^{8}$. Bien connue des peintres, cette technique de la "graticulation ", avait été présentée par Albrecht Dürer dans une planche de son livre sur les proportions et les mensurations. Elle permettait de "rapporter les parties renfermées en chaque carreau, dans d'autres carreaux proportionnés ${ }^{9}$ et d'obtenir une image bidimensionnelle à partir de modèles tridimensionnels complexes. Pour cette raison, elle avait parfois été employée pour illustrer des traités d'anatomie : ce fut le cas des dessins réalisés par Jan Wandelaar pour celui de Bernhard Siegfried Albinus, dont Camper avait d'ailleurs suivi les leçons. Il s'agissait par ce moyen de concilier des vues rapprochées du corps avec le respect scrupuleux des proportions, ce qui n'était possible qu'en évitant les raccourcis et les distorsions de perspective.

Fig. 2. Petrus Camper, «La ligne facial du type européen et de l'Apollon », Dissertation physique sur les différences que présentent les traits du visage, Utrecht, 1791.

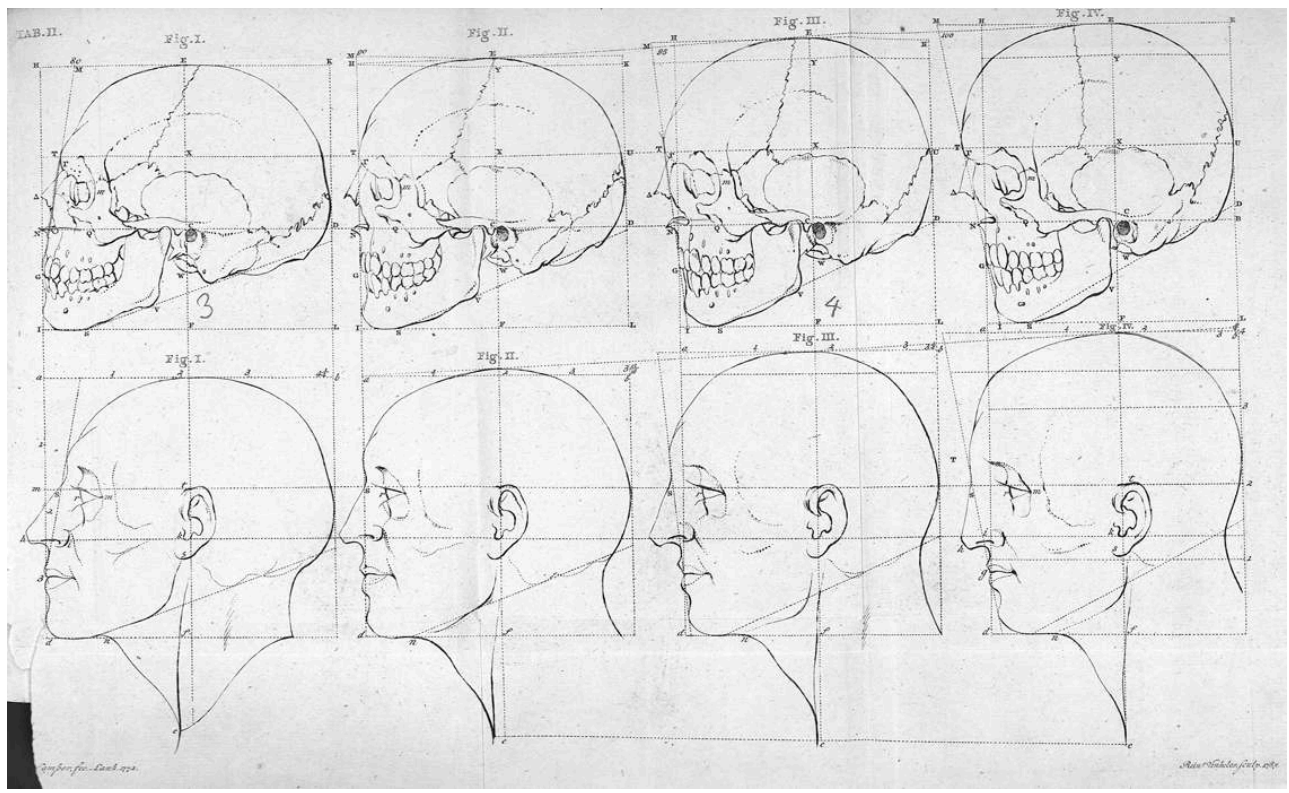

Photo : Bibliothèque interuniversitaire de médecine.

4 Au moment où les théories de Camper pénétrèrent en France, les procédés mécaniques du dessin appliqués à la reproduction des traits humains connaissaient une faveur sans précédent. On sait l'engouement de l'époque pour le trait de contour et les profils découpés en ombre chinoise ${ }^{10}$, cette forme de portrait épuré où la main de l'artiste intervenait peu, mais que Johann Kaspar Lavater prisait par-dessus tout. Sa célèbre "machine à tirer des silhouettes" montre que lorsqu'elles étaient appliquées aux représentations du corps humain, la rationalité technologique et l'abstraction linéaire relevaient autant du champ artistique que de la connaissance de l'homme comme objet physique, moral et intellectuel. Ce que confirme, à la même époque, l'apparition d'un autre appareil, le "physionotrace", au moyen duquel on exécutait des portraits de profil avec une économie de dessin et une tendance à l'épuration plus propice encore aux classements typologiques. Mis au point en 1786 par le graveur et musicien GillesLouis Chrétien, il se présentait comme une sorte de grand pantographe placé sur un plan vertical : à partir d'un système de parallélogrammes articulés, il permettait à l'opérateur de suivre les contours de son modèle à travers un œilleton, tandis qu'un 
stylet les reproduisait simultanément sur une feuille de papier. Avec pour résultat un profil grandeur nature, pratiquement sans déformation perspective, soit l'équivalent d'un buste en projection géométrale.

Fig. 3. Johann Kaspar Lavater, "Machine sûre et commode pour tirer les silhouettes ", L'art de connaître les hommes par la physionomie par Gaspar Lavater, nouvelle édition augmentée par Moreau (de la Sarthe), Paris, Depelafol, 1807.

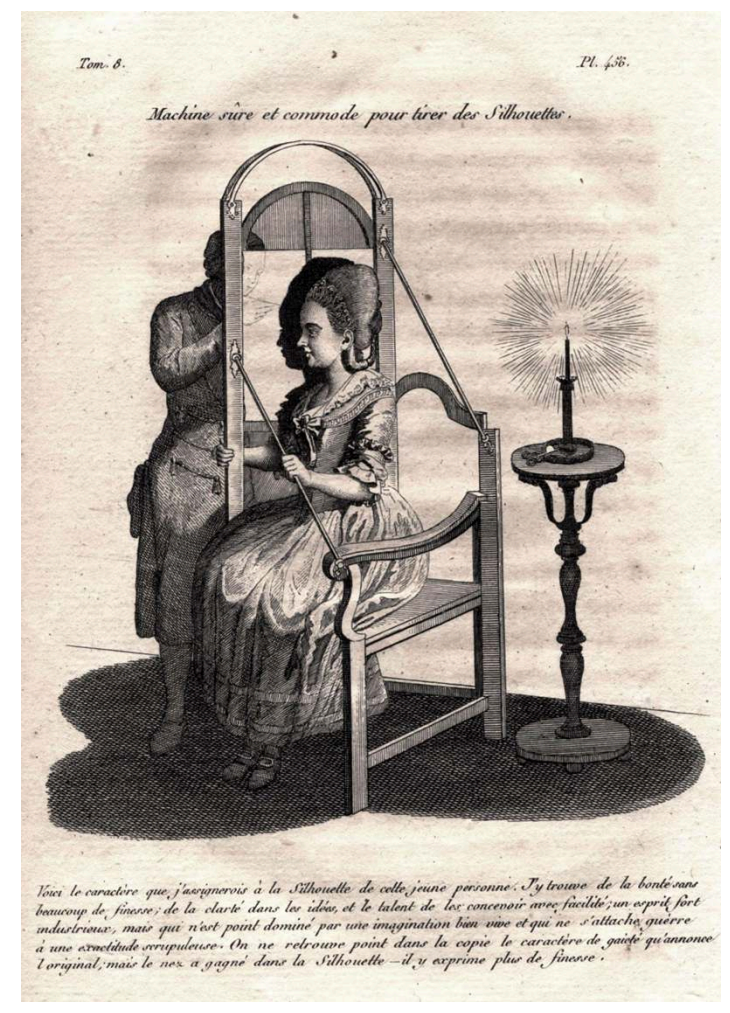

Photo : Martial Guédron

5 Avant de revenir plus directement aux illustrations de Camper, quelques remarques s'imposent à propos de ces appareils. En effet, s'ils ont contribué à la démocratisation $\mathrm{du}$ portrait, ils ont aussi été appréciés pour leurs applications dans le domaine indiciaire ou judiciaire. Sur les avantages de la silhouette, Lavater est intarissable :

«Les silhouettes seules ont étendu mes connaissances physiognomoniques plus que tout autre portrait; elles ont exercé mon sentiment physiognomonique, plus que la contemplation même de la nature toujours variée et jamais uniforme. La silhouette fixe l'attention: en l'arrêtant aux seuls contours extérieurs, elle simplifie l'observation, qui devient par là plus facile et plus exacte; je dis l'observation, et par conséquent aussi la comparaison. La silhouette est une preuve positive et incontestable de la réalité de la science des physionomies ${ }^{11}$. "

Quant au physionotrace, on avait estimé qu'il aiderait à fixer les traits des conscrits et à repérer les déserteurs ${ }^{12}$. On comprend mieux, à partir de là, que le principe en ait été adapté à certains dispositifs conçus par les premiers anthropologues. S'ils ne donnèrent aucune suite au châssis quadrillé de Camper, leur recours au diagraphe, au daguerréotype et surtout à toutes sortes d'instruments qui permettaient d'effectuer des mesures précises à partir de crânes en projections latérales n'est pas sans filiation avec l'emploi des machines à silhouetter ${ }^{13}$. On soulignera ici que dans les discours sur l'art, en particulier ceux qui se rapportaient à la théorie de l'imitation de la nature, toutes 
ces pratiques se situaient aux antipodes de ce qu'on attendait des artistes véritables ; à l'évidence, elles relevaient d'une imitation mécanique et sans âme. C'est pourquoi dans le langage des ateliers, des appareils à dessiner comme le pantographe étaient parfois appelés des « singes $»^{14}$.

\section{Échelles et hiérarchies}

7 À partir de 1791, les théories de Camper furent directement accessibles au lectorat français grâce à deux traductions simultanées de ses écrits, celle du libraire parisien d'origine hollandaise Hendrik Jansen et celle de Denis-Bernard Quatremère d'Isjonval, l'excentrique aîné de Quatremère de Quincy. On y apprenait que l'angle facial était déterminé par la jonction de deux plans, l'un passant par le conduit auditif externe et l'épine nasale, l'autre par l'épine nasale et la partie la plus saillante du front. Camper entendait prouver ainsi que plus le front s'élevait perpendiculairement et moins les mâchoires faisaient saillie en avant, plus l'angle facial était ouvert, tandis que plus le front était incliné en arrière et les mâchoires s'allongeaient en avant, plus cet angle était aigu. Sa démonstration reposait autant sur des spéculations anthropologiques que sur des préventions esthétiques. Non seulement il ne cessait de faire référence à des ouvrages traitant de la théorie et de la pratique du dessin, mais il se flattait, grâce à son procédé, d'offrir aux artistes le moyen le plus utile et le plus sûr de rendre compte des différences de physionomies entre les peuples ${ }^{15}$. À dire vrai, ses schémas de crânes ont fini par imposer l'idée d'une hiérarchie des types humains compris entre le singe et l'Apollon du Belvédère. Bien entendu, le type européen se trouvait placé du côté du dieu grec et le type africain du côté du singe, un animal dont Camper ne pouvait ignorer qu'il était un vieux symbole de l'homme sans âme, une créature certes fascinante, mais réifiée dans les pulsions corporelles et d'une laideur physique traditionnellement perçue comme un indice de laideur morale.

8 Avec la planimétrie, le recours au modèle archétypal fourni par l'Apollon du Belvédère prouve que Camper adhérait parfaitement aux options stylistiques de la fin du xvIII ${ }^{\mathrm{e}}$ siècle. Celles-ci ont fortement pesé sur ses critères anthropologiques puisqu'il a réservé la place de choix au type européen, gratifié d'un angle facial de 80 degrés, celui du dieu grec ayant une valeur absolue approchant les 100 degrés, tandis que les types négroïdes n'offraient selon lui qu'une ouverture de 70 degrés. Certes, Camper ne doit pas être regardé comme le père fondateur du racisme biologique. Dans les explications qu'il fournit de ces schémas, il avertit d'ailleurs le lecteur qu'il ne faudrait surtout pas tirer de conclusions hâtives de quelques analogies apparentes entre les crânes des Africains et ceux des singes ${ }^{16}$. Par ailleurs, il sait que les angles faciaux supérieurs à 80 degrés ne se trouvent pas dans la nature. Pourtant, si la physiognomonie raciale ne s'inspire guère des théories qu'il a développées, ses célèbres illustrations ont fini par accréditer le principe d'une équation entre la structure du crâne, le volume du cerveau et les qualités de l'esprit. À cet égard, on relèvera en quels termes Georges Cuvier et Étienne Geoffroy Saint-Hilaire se sont approprié l'angle de Camper, tout en prétendant le doter de repères géométriques plus rigoureux :

«[...] La grandeur et la convexité du crâne indiquent la sensibilité, comme le prolongement et la grosseur du museau indiquent la brutalité. On observe dans les diverses races d'homme la même série de rapports, que dans les diverses espèces d'animaux, entre la saillie du crâne et le degré d'intelligence ou de cette sensibilité 
exquise, de cette mobilité dans les organes qui fait peut-être la principale base des

différences qui existent d'homme à homme ${ }^{17}$ ».

Dix ans plus tard, apportant des précisions sur ces questions, Cuvier ferait lui aussi de la statuaire antique une référence absolue. À l'en croire, c'était parce que les artistes de l'Antiquité avaient parfaitement compris que la ligne faciale relevée était un signe de compétence morale et intellectuelle, mais aussi un des caractères de la beauté, qu'ils l'avaient inclinée davantage que ce qu'on peut trouver dans la nature, cela afin de les opposer significativement aux êtres imparfaits et aux brutes ${ }^{18}$.

Fig. 4. Perot, « Le stéréographe de Broca construit par Alfred Molteni ».

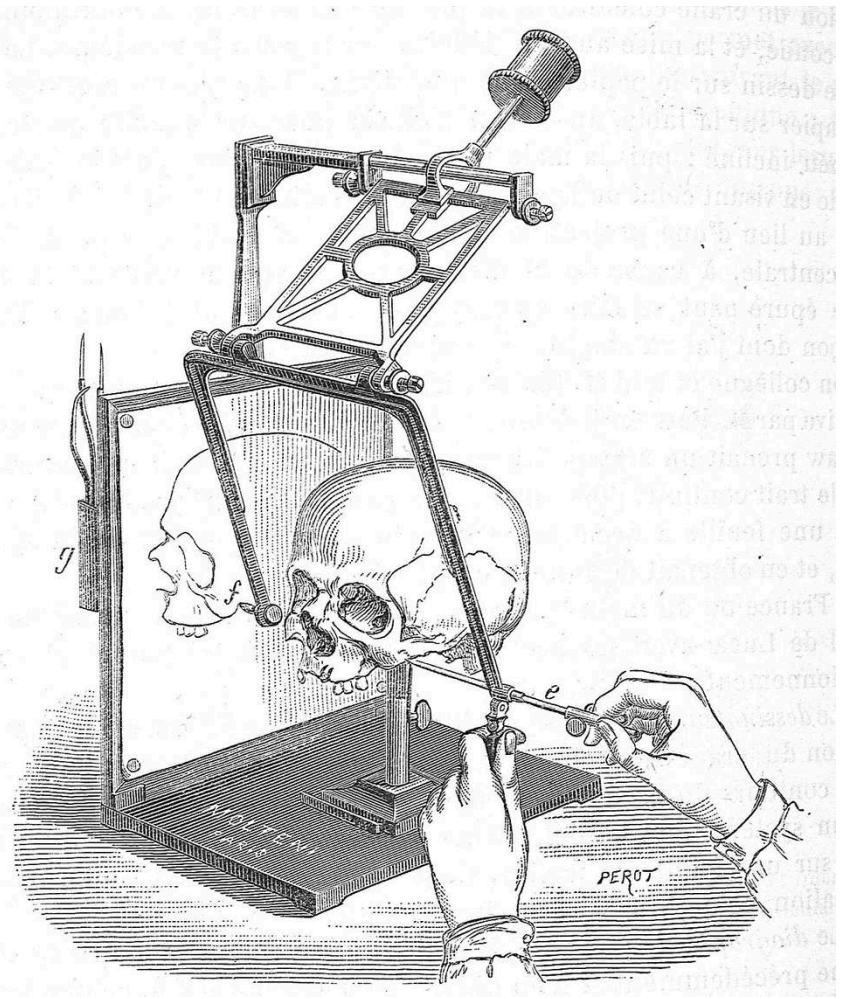

Photo : Martial Guédron

Comme l'a noté son contemporain l'anatomiste écossais Charles Bell, les ouvrages de Camper prouvent qu'il s'est également fondé, pour ses démonstrations, sur l'observation de gemmes, de camées et de médailles anciennes dans lesquels il a trouvé différents types physiques rigoureusement inscrits de profil ${ }^{19}$. De fait, s'il a émis des réserves sur les illustrations réalisées par le comte de Caylus pour les volumes de son Recueil d'Antiquités, Camper ne s'est pas privé d'en tirer des remarques sur le visage des nègres, en particulier à partir de transpositions gravées de vases et de lampes anthropomorphes aux profils caricaturaux ${ }^{20}$. Cette prééminence du profil, considéré comme le meilleur moyen de saisir les différences de morphologies entre les visages, ne s'est pas démentie par la suite : elle a même fini par imposer un clivage entre l'avant et l'arrière, autrement dit entre la race frontale des blancs, censée se distinguer par ses facultés mentales élevées, et la race occipitale des nègres, prédisposée à laisser libre cours à ses sensations et à ses passions. Plus ou moins réglées sur le modèle grec, ces grilles de lecture ont d'ailleurs permis d'adapter la vieille hiérarchie entre le haut et le bas du visage à la nouvelle segmentation du genre humain. Pour aller vite, l'ampleur du 
front et l'éclat des yeux étaient le signe de l'activité mentale, alors que le développement des lèvres, des mâchoires et des dents renvoyait au digestif et au sexuel, sinon à la violence et aux pulsions, toutes choses censées caractériser le nègre et stigmatiser sa nature profonde. Les propos de Sonnini de Manoncourt, naturaliste héritier de Buffon et membre de la Société des observateurs de l'homme, paraissent sans appel :

«Ceux [parmi les hommes] qui ont une figure avancée en museau montrent un air ignoble et bête, au lieu que les visages droits, avec une bouche rentrante et un front saillant, présentent une figure intelligente, noble et majestueuse ; aussi les anciens sculpteurs grecs, dont les ouvrages sont si admirables, ne représentoient jamais leurs dieux qu'avec cet attribut de suprême intelligence et de génie. Ils ouvraient beaucoup l'angle facial, et lui donnaient même 90 à 100 degrés. Dans Jupiter, le dieu suprême, le front s'avance extraordinairement; il semble grossi de l'éternelle sagesse, et rempli des destinées de l'univers. Bien loin d'offrir un angle saillant, la face du dieu est rentrante, ce qui lui donne un air de sublimité concentrée et de réflexion convenable au père de la nature, des dieux et des hommes ${ }^{21}$."

11 Ce clivage radical entre le haut et le bas, parfaitement mis en lumière dans les ouvrages à visée encyclopédique et utilitaire, est indissociable de certaines images apparues dans les mêmes années. En raison de leur ordonnancement horizontal, les célèbres illustrations de Camper semblaient impliquer une évolution qui, dans un sens, entraînait la race blanche vers sa perfection, dans l'autre, vers sa dégénération. Là encore, les débats sur ces questions témoignent de l'immixtion des préjugés esthétiques dans les taxinomies anthropologiques: si l'on acceptait le point de vue suivant lequel le monde sauvage était un monde enfant, ne pouvait-on dire que ses représentants étaient en quelque sorte des "ébauches", presque dans le sens néoclassique du terme, de ce que la race blanche accomplissait en tendant vers la pureté stylistique du modèle antique? Comme les artistes, la nature ne procédait-elle pas par tâtonnements et par approximations avant d'accomplir sa création la plus parfaite ${ }^{22}$ ? 
Fig. 5. Petrus Camper, «Différents profils d'après des médailles antiques », Dissertation physique sur les différences que présentent les traits du visage, Utrecht, 1791.

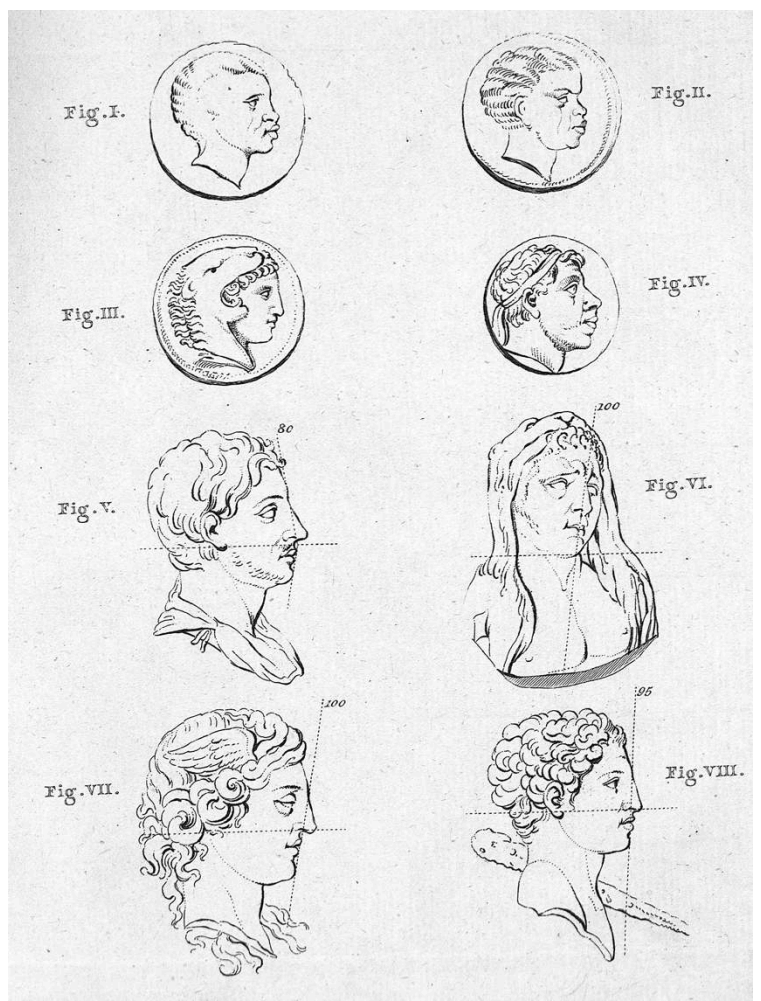

Photo : Bibliothèque interuniversitaire de médecine.

\section{La nature, l'idéal et la caricature}

En cette période qui se passionnait pour toutes les formes de croisement entre les différentes espèces vivantes, les schémas de Camper avaient renforcé la menace d'une contiguïté de l'homme et de la bête, contiguïté que laissait déjà pressentir la première édition du Systema naturæ de Carl von Linné en $1735^{23}$. La suite de l'article de Sonnini vaut d'être largement citée :

«La race nègre a toujours paru très-inférieure en esprit aux autres races, et quelque soin qu'on ait pris d'instruire de jeunes nègres, on ne voit pas qu'ils aient produit quelque homme de grand génie. D'ailleurs, cette soumission à l'esclavage annonce peu de vigueur dans le caractère et peu d'élévation dans l'esprit. La nature a maltraité ces pauvres nègres en ne leur donnant ni assez de courage ni assez d'intelligence ; ils seront toujours dans la servitude par foiblesse, et dans la barbarie par impuissance à réfléchir. Nous en voyons une marque dans leur visage prolongé en museau et leur front abaissé. Si les organes de l'appétit dominent chez eux sur ceux de la pensée, nous l'observons de même dans leurs affections qui tiennent plus $\mathrm{du}$ physique que du moral. À cet égard, le Hottentot l'emporte encore sur le nègre ; c'est presque le premier des orangs-outangs, car son ame [sic] est si bien cachée dans la matière du corps, qu'on y trouve pour ainsi dire que l'animalité ${ }^{24}$. »

En l'an II, devant la Convention nationale, l'abbé Grégoire avait proposé de regarder l'homme civilisé comme un homme dégénérée ${ }^{25}$. Mais il est facile de comprendre que la proximité de l'homme et de l'animal paraissait moins dérangeante lorsque le processus de dégénération était interprété en sens inverse: l'influent Johann Friedrich Blumenbach lui-même n'avait-il pas suggéré, après Maupertuis, que la 
couleur blanche était la couleur originaire et qu'il était plus facile à un blanc de devenir un homme de couleur, qu'à un homme de couleur de devenir un blanc ${ }^{26}$ ? Au surplus, faire du nègre une figure dégradée du blanc servait à conforter les visées esclavagistes, voire à légitimer l'idée d'en présenter quelques spécimens dans des zoos humains. Si l'on pouvait aller jusqu'à admettre que même les formes humaines les plus viles dérivaient de la souche la plus élevée, l'homme blanc était évidemment considéré comme l'archétype, la norme et donc le spécimen le plus beau. Pour les disciples de Buffon et de Blumenbach, tout comme pour ce grand lecteur de Buffon qu'était Winckelmann, le facteur mésologique expliquait largement ce processus: la dégradation avait conduit certains représentants de l'espèce humaine à s'écarter de leur beauté originelle au point d'évoquer l'orang-outang, le satyre des anciens et tous les autres hominidés incertains. Cela dit, le tournant $d u$ xvIII $^{\mathrm{e}}$ siècle vit aussi l'émergence d'une anthropologie raciale qui, cette fois, allait refuser d'interpréter la diversité humaine sous le mode du continuum et substituer les explications essentialistes aux théories environnementalistes. C'est là que le racisme biologique a trouvé son socle, avec la nouvelle définition de la race entendue comme ensemble des caractères naturels permanents, une définition que Kant aurait bien du mal à concilier avec le principe de l'unicité de l'espèce humaine ${ }^{27}$.

Fig. 6. Anne-Claude-Philippe de Caylus, Recueil d'antiquités Égyptiennes, Étrusques, Grecques, Romaines, et Gauloises, Paris, N. M. Tilliard, t. V, 1762, planche XC, fig. 2.

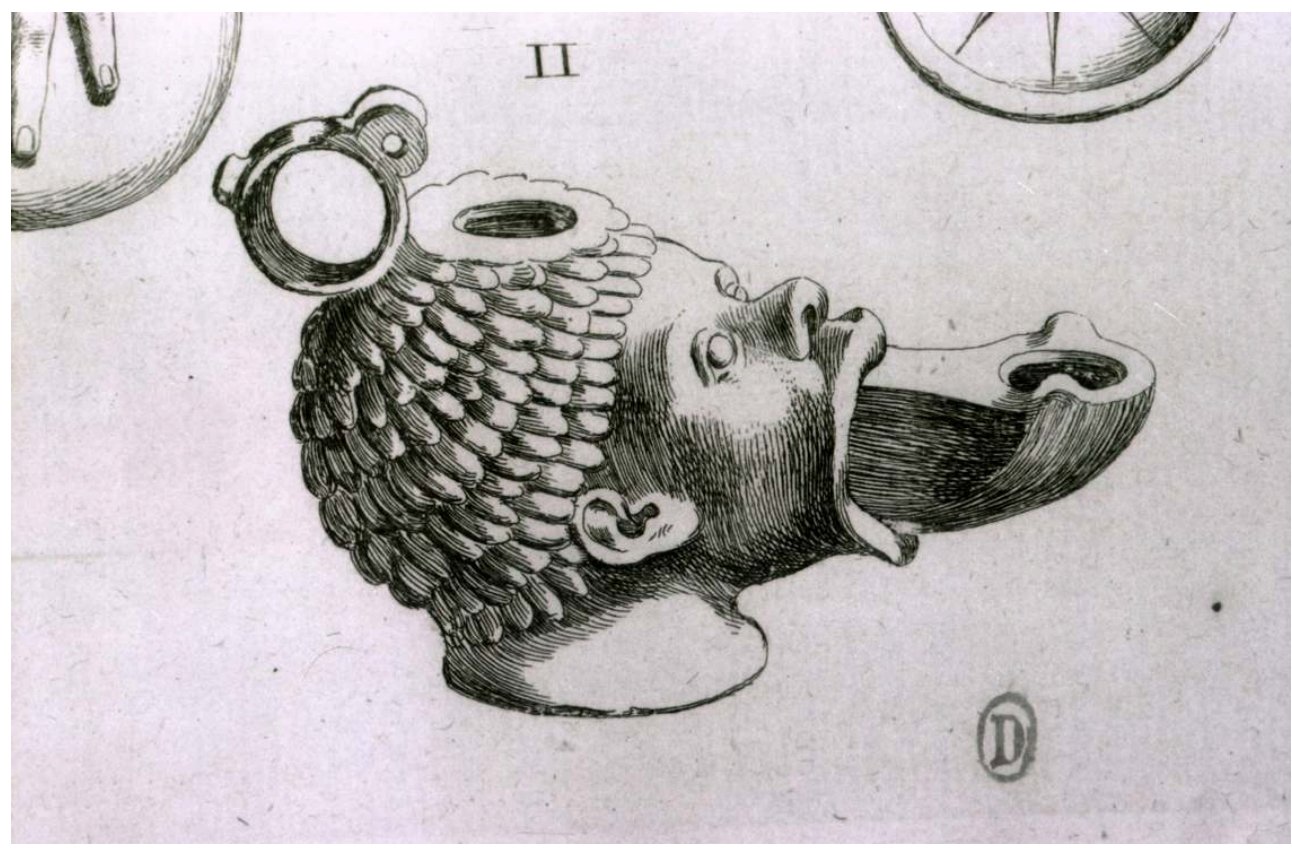

Photo : Bibliothèque de l'Institut national d'histoire de l'art. Collections Doucet.

Pour autant, les préjugés esthétiques continueraient largement d'interférer dans les théories de la pluralité des races. En témoignent les écrits de Julien-Joseph Virey pour qui le nègre était tout à l'opposé de l'Européen :

«L'homme noir est né imitateur, comme le singe; il reconnaît la supériorité intellectuelle du blanc, supporte assez aisément son esclavage, est très insouciant et paresseux. Ces habitudes annoncent une mollesse naturelle ou innée de l'âme. [...] À mesure que les mâchoires se rapetissent, le front s'avance, le cerveau s'étend et s'agrandit, le naturel prend plus d'énergie, et l'âme plus d'activité. Il suit de là que 
le nègre est en quelque sorte l'inverse de l'Européen, par la forme et la capacité de

son crâne signalant la faiblesse et la dégradation de son âme. ${ }^{28}$ "

Lorsqu'on met en relation ces affirmations lapidaires avec les planches gravées qui accompagnent ses écrits, on comprend que chez Virey, l'équation entre dissimilitude formelle et discrimination ontologique s'articule sur un rapport dialectique entre le modèle et le contre-modèle. Dans son Histoire naturelle du genre humain, le modèle a d'abord été figuré par la tête de l'Apollon du Belvédère vue de profil, puis, dans les éditions suivantes, par celle du Jupiter Vérospi du Vatican. Quant au contre-modèle, il a toujours été représenté par l'orang-outang et le nègre, leur proximité, pour Virey, ne faisant aucun doute : comme nombre de ses confrères, il assimilait le visage négroïde à un museau et la race qu'il fixait au niveau le plus bas de l'humanité était celle qui incarnait pour lui la plus grande laideur. À cet égard, ses jugements sur la question n'étaient pas seulement tributaires de Maupertuis ou de Buffon, mais aussi des illustrations diffusées dans l'espace savant depuis un demi-siècle. Souvent, Virey donne le sentiment qu'il évalue les êtres vivants comme s'il s'agissait d'images. Au reste, lorsqu'il suggère qu'à l'époque où les Grecs présentaient les formes les plus parfaites, les nègres personnifiaient déjà l'envers du beau, il se réfère, comme Camper avant lui, aux estampes du Recueil d'Antiquités du comte de Caylus ${ }^{29}$. Ce choix n'a rien de hasardeux : Caylus faisait partie de cette nouvelle génération d'«antiquaires» qui accordaient davantage de crédit aux médailles, aux gemmes et finalement à tous les objets pouvant être représentés par des images, qu'aux discours et aux interprétations philologiques de leurs prédécesseurs ${ }^{30}$.

Il est significatif que cette collusion entre les critères anthropologiques et les critères esthétiques se vérifie au même moment dans certaines images didactiques à destination des artistes. Mais il convient surtout de souligner ici qu'à la différence des schémas de Camper, nous avons maintenant affaire à une hiérarchisation qui se fait selon une échelle verticale, avec dans certains cas une fusion entre le représentant de la race blanche et le modèle fourni par les statues antiques : ignorant qu'elles avaient perdu les couleurs qui les décoraient autrefois, on associait leur monochromie à leur pureté et à leur noblesse. En interaction avec les textes, ces images suggèrent qu'à l'instar de l'orang-outang ou du singe en général, le nègre n'est qu'une imitation sans âme, une sorte de grimace de l'homme blanc. 
Fig. 7. Duhamel, « l'angle facial des espèces », in Julien-Joseph Virey, Histoire naturelle du genre humain, Paris, 1801, t. 2.

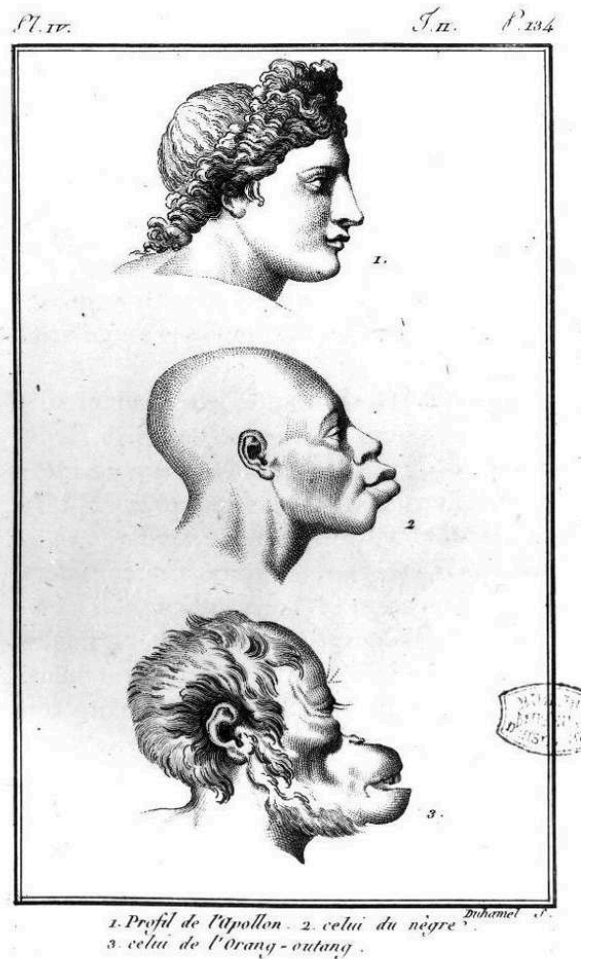

Photo : Bibliothèque interuniversitaire de médecine.

Cette hiérarchisation des représentants de l'espèce humaine prend un sens plus précis lorsqu'on la relie à la question récurrente du discours sur l'art au siècle des Lumières, celle de l'imitation de la nature. Parmi les nombreuses sources anciennes qui étaient convoquées dans ces débats, il faut revenir au fameux passage de la Poétique (II, 1448a) où Aristote évoque les trois styles caractérisant les trois peintres majeurs de l'Antiquité : Polygnote, Dionysos et Pauson. D'après le philosophe athénien, Polygnote représentait ses modèles en mieux, Dionysos à l'identique et Pauson en pire. À la période qui nous occupe, l'imitation du modèle « en mieux » correspondait à l'imitation "idéalisante », celle qui prétendait corriger les défauts de la nature pour tendre vers la perfection du modèle antique. Symétriquement, l'imitation « en pire » correspondait à la caricature, qui connaissait alors des développements considérables. En d'autres termes, si les représentants des races inférieures s'apparentaient à des caricatures, c'est qu'à l'instar des singes, ils étaient des imitations à rebours par rapport aux normes corporelles établies. C'est déjà comme des êtres caricaturaux que Buffon regardait les Kalmouks, dont il faisait les représentants les plus laids de tous les hommes ${ }^{31}$. Que les Hottentots aient eu le triste privilège de leur succéder, devenant des caricatures de nègres, ne change rien à l'affaire. Restait la troisième possibilité suggérée par Aristote, l'imitation à l'identique, qui aboutissait à des types physiques sans particularités individuelles, ne tenant ni de l'idéalisation, ni de l'exagération des signes distinctifs. C'est de là, peut-être, qu'allait émerger la nouvelle norme qui, pour les polygénistes, masquerait les hommes hétérogènes, celle de l'homme moyen cher au statisticien belge Adolphe Quételet. Dès lors, privilégiant le quantitatif sur le qualitatif, on se mettrait en quête du crâne type, produit des nombres et moyenne de toutes les têtes. 


\section{BIBLIOGRAPHIE}

Bell 1904 : Charles Bell, The Anatomy And Philosophy of Expression as connected with he Fine Arts, Londres, George Bell \& Sons, 1904.

Blanckaert 1987 : Claude Blanckaert, «Les Vicissitudes de l'angle facial et les débuts de la craniométrie (1765-1875)», Revue de synthèse, 1987, IVe série, nº 3-4, p. 417-453.

Blanckaert 2000 : Claude Blanckaert, « 1800. Le moment "naturaliste" des sciences de l'homme », Revue d'histoire des sciences humaines, 2000, n 3, p. 117-160.

Blumenbach 1804 : Johann Friedrich Blumenbach, De l'unité du genre humain, et de ses variétés..., Paris, Allut, 1804 ; trad. du latin : Frédéric-Charles Chardel.

Broca 1860-1863 : Paul Broca, « Mémoire sur le crâniographe ", dans Mémoires de la Société d'anthropologie de Paris, Paris, Victor Masson \& Fils, 1860-1863, t. I, p. 373.

Buffon 1777 : Georges-Louis Leclerc, comte de Buffon, Histoire naturelle, générale et particulière, servant de suite à l'histoire des animaux quadrupèdes, Paris, 1777.

Camper 1791 : Pierre Camper, Dissertation physique de M. Pierre Camper, sur les différences réelles que présentent les traits du visage chez les hommes des différents pays et des différents âges, sur le beau qui caractérise les statues et les pierres gravées, Adrien-Gilles Camper, éd., Utrecht, B. Wild \& J. Altheer ; trad. fr. : Denis-Bernard Quatremère d'Isjonval, 1791.

Camper 1803 : Pierre Camper, «Éloge de Pierre Camper par Condorcet », dans Euvres de Pierre Camper qui ont pour objet l'histoire naturelle, la physiologie et l'anatomie comparée, Paris, H. J. Jansen, an XI [1803], vol. I.

Certeau, Julia et Revel 1976 : Michel de Certeau, Dominique Julia et Jacques Revel, Une politique de la langue. La Révolution française et les patois : l'enquête de Grégoire, Paris, Gallimard, 1976.

Cuvier 1805 : Georges Cuvier, Leçons d'anatomie comparée, Paris, Baudouin, 1805.

Cuvier et Geoffroy Saint-Hilaire 1795 : Georges Cuvier et Étienne Geoffroy Saint-Hilaire, « Histoire naturelle des orangs-outangs, des caractères qui peuvent servir à distinguer les singes », dans Magasin encyclopédique, 1795, t. III, p. 457.

D'un regard l'autre... 2006-2007 : D'un regard l'autre. Histoire des regards européens sur l'Afrique, l'Amérique et l'Océanie (cat. expo., Paris, musée du quai Branly, 2006), Yves Le Fur éd., Paris, RMN, 2006.

Décultot 2000 : Élisabeth Décultot, Johann Joachim Winckelmann. Enquête sur la genèse de l'histoire de l'art, Paris, PUF, 2000.

Gombrich (1971) 1987 : Ernst Hans Gombrich, L'Art et l'Illusion. Psychologie de la représentation picturale (1971), Paris, Gallimard, 1987 ; trad. fr. : Guy Durand.

Gossiaux 1985 : Pol-Pierre Gossiaux, « Anthropologie des Lumières (culture "naturelle” et racisme rituel) », dans Daniel Droixhe et Pol-Pierre Gossiaux, L'Homme des Lumières à la découverte de l'autre, Bruxelles, éd. de l'Université de Bruxelles, 1985.

Hennequin 1926-1927 : René Hennequin, Un photographe de l'époque de la Révolution et de l'Empire. Edme Quenedey des Riceys. Portraitiste du physionotrace, Troyes, Impr. J.-L. Platon, 1926-1927. 
Lavater (1807) 1820 : Gaspar Lavater, L'Art de connaître les hommes par la physionomie, Paris, éd. Depelafol, (1807) 1820.

Meijer 1999 : Miriam-Claude Meijer, Race and Aesthetics in the Anthropology of Petrus Camper (1722-1789), Amsterdam-Atlanta, Rodopi, 1999.

Moreau de Maupertuis (1745) 1768 : Pierre Louis Moreau de Maupertuis, Vénus physique. Euvres de Maupertuis, Lyon, Bruyset, (1745) 1768.

Pernety 1757 : Antoine-Joseph Pernety, Dictionnaire portatif de peinture, sculpture et gravure, avec un traité pratique des différentes manières de peindre, Paris, Beauche, 1757.

Quatremère de Quincy 1791 : Antoine-Chrysostome Quatremère de Quincy, Considérations sur les arts du dessin en France, suivies d'un plan d'Académie, ou d'École publique, et d'un système d'encouragement, Paris, Desenne, 1791.

Robinet 1768 : Charles Robinet, Considérations philosophiques de la gradation naturelle des formes de l'être ou les essais de la nature qui apprend à faire l'homme, Paris, Charles Saillant, 1768.

Schnapp 1993 : Alain Schnapp, La Conquête du passé. Aux origines de l'archéologie, Paris, éd. Carré, 1993.

Sonnini de Manoncourt 1804 : Charles-Nicolas-Sigisbert Sonnini de Manoncourt, Nouveau dictionnaire d'histoire naturelle, Paris, Déterville, an XII [1804].

Tinland (1968) 2003 : Franck Tinland, Homo ferus et Homo sylvestris : de l'animal à l'homme, Paris, L'Harmattan, (1968) 2003.

Virey 1800 : Julien-Joseph Virey, Histoire naturelle du genre humain, Paris, F. Dufart, 1800.

Virey 1824 : Julien-Joseph Virey, Histoire naturelle du genre humain, nouvelle édition augmentée et entièrement refondue avec figures, Paris, Crochard, 1824.

Winckelmann 1789 : Johann Joachim Winckelmann, Histoire de l'art chez les Anciens, Paris, Barrois \& Savoye, 1789.

\section{NOTES}

1. Gombrich (1971) 1987, p. 297 et 392.

2. Quatremère de Quincy 1791, p. 128.

3. Winckelmann, 1789 , t. II, p. 32-33 et 124.

4. Décultot 2000, p. 208-209.

5. Blanckaert 2000, p. 117-160.Voir également D’un regard l'autre... 2006-2007, en particulier la section 2, p. 104-141.

6. Meijer 1999, p. 21.

7. Voir Camper 1803, vol. I, p. XCIII.

8. Camper 1791, p. 36-37.

9. Pernety 1757 , p. 346.

10. Camper fait d'ailleurs référence au fameux mythe de l'invention du dessin par Dibutade, la fille d'un peintre potier de Sycone, qui aurait tracé sur un mur le contour de l'ombre de son amant pour en conserver l'image : Camper 1791,p. 93.

11. Lavater 1807 , t. VIII, p. 4.

12. Hennequin 1926-1927, p. 163.

13. Voir les réserves de Broca à l'encontre de ces appareils : Broca 1860-1863, t. I, p. 373. 
14. En 1762, dans sa quatrième édition, le Dictionnaire de l'Académie française entérine cet usage : «On appelle Singe, un instrument qui sert à copier mécaniquement des desseins, des estampes, sans aucune connoissance du dessein ou de la gravure. On l'appelle aussi Pantographe. »

15. Blanckaert 1987, p. 417-453.

16. Camper 1791, p. 35.

17. Cuvier et Geoffroy saint Hilaire 1795, t. III, p. 457.

18. Cuvier 1805, vol. 2, p. 162.

19. Bell 1904, p. 24-25.

20. Camper 1791,p. 3 et 40.

21. Sonnini de Manoncourt 1804, t. XXIII, p. 357, article « Visage et physionomie ».

22. Voir notamment Robinet 1768, p. 5.

23. Tinland (1968) 2003, p. 235-236.

24. Tinland (1968) 2003, p. 358.

25. Certeau, Julia et Revel 1976, p. 303.

26. Blumenbach 1804, p. 20. Voir Moreau de Maupertuis (1745) 1768, t. II, p. 125-128.

27. Gossiaux 1985, p. 56-57.

28. Virey 1824, p. 4-5.

29. Virey 1800 , t. 1, p. 150 , note 1.

30. Schnapp 1993, p. 295-296.

31. Buffon 1777 , t. IV, p. 486-487.

\section{RÉSUMÉS}

La charnière $\mathrm{du} 18^{\mathrm{e}}$ et $\mathrm{du} 19^{\mathrm{e}}$ siècles constitue une période privilégiée pour le chercheur qui s'intéresse aux confluences entre l'histoire de l'art et l'anthropologie : alors que d'une part de nombreux savants établissaient leurs systèmes de classification des peuples sur des critères esthétiques tels que la forme et la couleur, d'autre part des auteurs recommandaient aux artistes de s'inspirer davantage des méthodes d'analyse des anthropologues. Mieux, c'est une étonnante porosité entre préjugés esthétiques, scientifiques et moraux que révèlent certains textes fondateurs de l'anthropologie au tournant des Lumières quand ils disqualifient les types physiques non-européens en s'inspirant de la description des œuvres les plus célèbres de la sculpture antique. On constate en effet que les taxinomies raciologiques de l'anthropologie physique se fondaient sur des préjugés esthétiques puisés dans l'histoire de l'art archéologisante et que les différentes variétés humaines y étaient perçues à travers des images très connotées. Marquées par la rationalité technologique du temps et un goût pour l'abstraction linéaire, cellesci favorisaient un idéal esthétique néoclassique qui ne cessait d'interférer dans les représentations de l'altérité. Aussi, les illustrations des traités d'anthropologie évoluaient-elles parfois dans des champs sémiologiques proches de ceux de la caricature, en particulier à travers l'utilisation de critères communs de la déviance.

\section{INDEX}

Mots-clés : anthropologie, caricature, néoclassicisme, physiognomonie, raciologie, taxinomie 


\section{AUTEUR}

\section{MARTIAL GUÉDRON}

Martial Guédron est professeur d'histoire de l'art à l'université de Strasbourg. Il étudie les modèles épistémologiques dans les représentations du corps idéal et de l'altérité radicale (De chair et de marbre. Imiter et exprimer le nu en France (1745-1815), Paris, Honoré Champion, 2003). Il a travaillé en collaboration avec Laurent Baridon sur l'impact des théories physiognomoniques aussi bien dans l'art savant qu'à travers l'image satirique (Corps et Arts. Physiologies et physionomies dans les arts visuels, Paris, L'Harmattan, 1999 ; Homme-Animal. Histoires d'un face à face, Adam Biro, 2004 ; L'art et l'histoire de la caricature, Paris, Citadelles \& Mazenod, 2006). Il a contribué au catalogue de l'exposition « Figures du corps. Une leçon d'anatomie » à l'École nationale supérieure des beaux-arts (Paris, 2008) et travaille actuellement avec l'équipe du musée des beaux-arts de Nancy à la préparation de l'exposition « Beautés Monstres » dont il est le commissaire scientifique avec Sophie Harent. 